\title{
Diffuse oesophageal leiomyomatosis in a young female: presenting as a large posterior mediastinal mass
}

\author{
HDS Pradeep', Dhammika Rasnayaka', Harsha Gamage ${ }^{2}$, Ramani Punchihewa ${ }^{1}$ \\ ${ }^{1}$ Department of Thoracic surgery and Pathology, National Hospital for Respiratory Diseases Welisara. \\ ${ }^{2}$ Department of Gastrointestinal surgery, Teaching Hospital, Karapitiya.
} Key words: Oesophageal leiomyomatosis, diffuse leiomy-
omatosis of the oesophagus.

\section{Introduction}

Diffuse oesophageal leiomyomatosis is a rare benign disorder of the oesophagus which is seen in children and young individuals. Oesophageal leiomyomas represents less than $0.6 \%$ of all oesophageal tumours. Symptomatic patients with dysphagia require surgical intervention with esophagectomy and creation of a Neo esophagus using gastric conduit $(1,2)$.

\section{Case report}

23yr old female in 3rd trimester of her first pregnancy was investigated for shortness of breath for 1 month duration. Other than the constitutional symptoms of pregnancy she didn't have significant upper gastrointestinal symptoms. On chest radiography she was found to have a large opacity involving the left hemi thorax. She has undergone elective caesarean section and contrast enhanced computerized tomography scan of the chest and abdomen was done. It was revealed to be a mass at posterior mediastinum arising from the oesophagus. On orogastroduodenoscopy there were no mucosal lesions but external compression of the oesophageal lumen from $30 \mathrm{~cm}$ up $t 038 \mathrm{~cm}$ from the incisor tooth was noted. Gastro oesophageal junction (G.O.J) was at $38 \mathrm{~cm}$ from incisor tooth and stomach was macroscopically normal. After routine pre-operative assessment and optimization she was planned for esophagectomy.

Left sided thoraco - laparotomy was done, after peritoneal survey oesophageal mobilization was done under direct vision with sharp and blunt dissection up to cervical part of the oesophagus. There was a large extra luminal mass arising from the wall of the oesophagus at G.O.J.

Stomach mobilization and duodenal kocherisation was done for the formation of the neo oesophageal conduit based on right gastroepiploic artery. Cervical part of the oesophagus

Correspondence: I.H.D Saman Pradeep

E-mail: samaniddagoda@gmail.comcom

Received: 04-02-2018 Accepted: 25-03-2018

(iD) https://orcid.org/0000-0003-3526-1684

DOI: http://doi.org/10.4038/sljs.v36i2.8515 was isolated and mobilized from the left side of the neck. Esophagectomy was done and proximal oesophageal end was biopsied and sent for frozen sectioning since there were submucosal nodules at upper end of the oesophageal cut end.

Oesophageal reconstruction was done with the stomach conduit and cervical anastomosis was performed. Naso jejunostomy tube was placed for enteral feeding and bilateral intercostal drains, abdominal drain and neck drains were placed. She had a protracted recovery at the intensive care unit complicated by elevated liver enzymes. On post-operative day 7 oral contrast study was performed which revealed intact cervical anastomosis without leakage. She was gradually established with oral feeding. Histopathology revealed diffuse oesophageal submucosal leiomyomatosis with large leiomyomata at lower end of the oesophagus.

\section{Discussion and conclusion}

Diffuse leiomyomatosis of the oesophagus is a rare entity with an unknown incidence seen in children and young adults which is characterized by multiple submucosal hamartomas within oesophageal wall. It is commonly seen in females with male to female ratio of 1:1.6 and commonly involves lower third of the oesophagus. It usually presents with upper

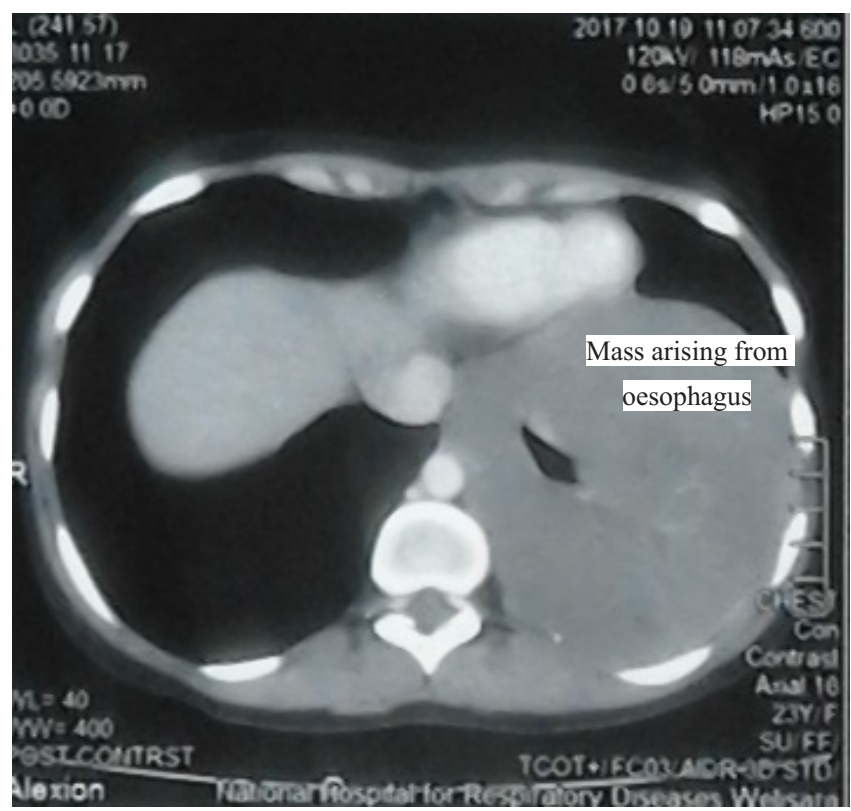

Figure 1. Contrast enhanced computed tomography (CECT) chest 


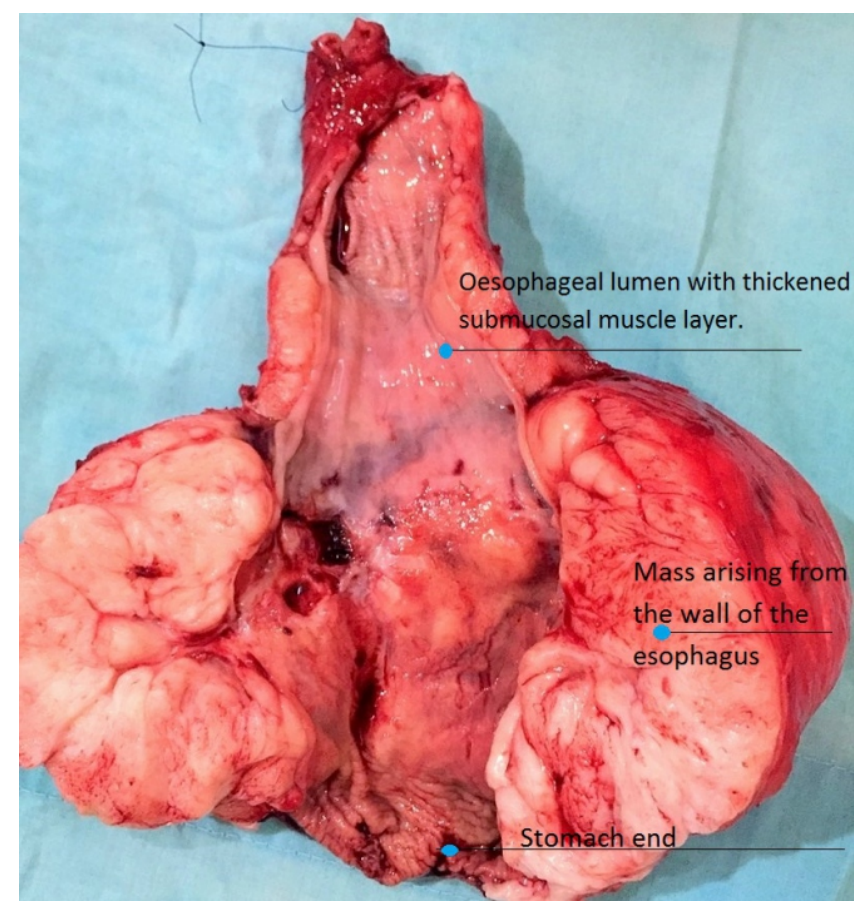

Figure 2. Esophagectomy specimen

gastrointestinal symptoms like dyspepsia, regurgitation and progressive dysphagia when it causes luminal compression.

It has a strong association with Alports syndrome and with leiomyomatosis of rest of the gastrointestinal tract and genitourinary tract (4). Histopathological analysis reveals smooth muscle proliferation with in the muscularis propria of the oesophagus results in multiple submucosal leiomyomata and they coalesce to form a large mass which occupies the posterior mediastinum with external compression of the oesophageal lumen.

Immunohistochemical analysis helps in differentiating the condition with gastro intestinal stromal tumours as it shows diffuse cytoplasmic immunoreactivity for smooth muscle actin (SMA) with no immnunoreactivity for CD117 and CD34 markers. In our case SMA was diffusely positive.

A symptomatic patient presents due to compression of the oesophageal lumen and may mimic oesophageal carcinoma or achalasia (3). Patients are incidentally diagnosed with routine chest $\mathrm{X}$ ray while on investigation for respiratory symptoms when it compresses lung parenchyma as a large mass occupying the posterior mediastinum as in our case.

Orogastroduodenoscopy, Barium swallow and contrast enhanced computerized tomography of the chest and abdomen are used to diagnose the condition.

Symptomatic patients with diffuse oesophageal involvement should undergo esophagectomy with neo oesophagus creation with a gastric conduit. When there is concomitant involvement of the stomach with leimyomata, colon or small bowel should be used for neo oesophagus creation. Due to the benign nature of the condition Patients who have undergone surgery has a good long term outcome. Young patients should be followed up in long term for renal, ocular and chochlea impairment to diagnose Alports syndrome in association with this condition. Genetic testing and counselling should be done in patients with a positive family history.

All authors disclose no conflict of interest. The study was conducted in accordance with the ethical standards of the relevant institutional or national ethics committee and the Helsinki Declaration of 1975, as revised in 2000 .

\section{References}

1. Lekawale H, Khandeparkar SS,Deshmukh S, Khadilkar A. A rare case of giant diffuse esophageal leiomyomatosis. Med J DY Patil Univ 2015;8:384-6. DOI: 10.4103/0975-2870.157095

2. Lee LS, Nance M, Kaiser LR, Kucharczuk JC.Familial massive leiomyoma with esophageal leiomyomatosis: an unusual presentation in a father and his 2 daughters. J Pediatr Surg. 2005 May;40(5):e29-32. DOI: 10.1016/j.jpedsurg.2005.02.016

3. Ray S, Saluja SS, Gupta R, Chattopadhyay TK. Esophageal leiomyomatosis - An unusual cause of pseudoachalasia. Can J Gastroenterol 2008;22:187-9. PMCID: PMC2659141

4. McKeeby JL, Li X, Zhuang Z, Vortmeyer AO, Huang S, Pirner M, Skarulis MC, James-Newton L, Marx SJ, Lubensky IA. Multiple leiomyomas of the esophagus, lung, and uterus in multiple endocrine neoplasia type .Am J Pathol.2001Sep;159(3):1121-7. doi: 10.1016/S0002-9440(10)61788-9.

\section{Learning Points:}

- Diffuse oesophageal leiomyomatosis is a rare benign disorder of the oesophagus which is seen in children and young individuals.

- It has a strong association with Alports syndrome and with leiomyomatosis of rest of the gastrointestinal tract and genitourinary tract. 